\title{
The nuclear receptor SF-1 (steroidogenic factor-1) is no longer an orphan
}

\author{
Jérôme Bertherat \\ Clinique des Maladies Endocriniennes et Métaboliques, Hôpital Cochin, 27 rue du Fabourgg-St-Jacques, 75014 Paris, France \\ (Correspondence should be addressed to J Bertherat)
}

The nuclear hormone receptor SF-1 (steroidogenic factor-1, also termed adrenal 4-binding protein or Ad4BP) is a major regulator of the development and function of the hypothalamic-pituitary-adrenal and -gonadal axes. It was initially discovered as a key transcription factor regulating the cytochrome $\mathrm{P} 450$ steroid hydroxylases in the gonads and the adrenal cortex $(1,2)$. The promoter of the genes regulated by SF-1 contains an AGGTCA-derived motif that binds SF-1. This nuclear receptor also regulates the expression of the steroidogenic acute regulatory protein, the adrenocorticotrophin receptor, and, in the pituitary, the $\alpha$-subunit of glycoproteins (3). Interestingly, SF-1 seems to play a role in cAMP regulation of transcription in the adrenal cortex.

In keeping with a major role for this nuclear receptor in a limited set of organs, SF-1 expression is regulated in a tissue-specific manner. It was initially reported to occur in the adrenocortical cells, ovarian theca and granulosa cells and in the testicular Leydig cells. Later, expression of SF-1 was also found in the ventromedial hypothalamic nucleus and in the pituitary gonadotrophs (4). Knockout mouse technology by targeted disruption of the SF-1 gene has clearly demonstrated the major role of SF-1 in development. All animals died within a week of birth from adrenocortical insufficiency that could be prevented by steroid hormone replacement therapy (5). The adrenal glands and the gonads were absent in the knockout mice, explaining the hormonal defect. Furthermore, a male-to-female sex reversal of external genitalia was observed, since all the knockout mice had female genitalia irrespective of genetic sex. Therefore, SF-1 also plays an important role in sexual differentiation. This last finding might be explained by SF-1 regulation of Müllerian inhibiting substance gene expression (6).

SF-1 belongs to the nuclear receptor family and shares conserved regions with the other members of this superfamilly. These regions are functional domains known to be important for these receptors. For example: two zinc fingers that mediate DNA binding, an AF-2 transactivation domain at the carboxyl terminus, a proline-rich domain that might mediate transactivation and a consensus site for protein kinase A phosporylation in response to cAMP pathway activation (see 7 for review).
Despite SF-1 homology with the nuclear hormone receptors, its ligand had not yet been identified, and it was not possible to know whether a steroid would modulate its activity. Lala and colleagues have now identified some oxysterols as SF-1 ligands that can stimulate SF-1-dependent transcription (8). They have tested several intermediates in the steroidogenic pathway to search for potential SF-1 ligands. In a transient transfection assay they have shown that the 25-, 26and 27-hydroxycholesterols specifically stimulate the activity of a truncated 21-hydroxylase promoter containing the SF-1 binding site. 25-Hydroxycholesterol is in fact the most potent stimulator of SF-1-dependent transcription. This activation is specific for SF-1 as other nuclear receptors do not seem to respond to these oxysterols. The AF-2 domain of the nuclear receptors is important for ligand-dependent transactivation. Deletion of the AF-2 domains of SF-1 dramatically reduces the transcriptional response to 25-hydroxycholesterol. This observation strongly suggests that oxysterols act on SF-1 as ligands. Interestingly, another member of the nuclear receptor family, LXR, has recently been shown to be regulated by oxysterol binding (9). However, transactivation seems to be quite specific, since 25-hydroxycholesterol is more effective at activating SF-1 than LXR, while 22-hydroxycholesterol strongly activates LXR but is less efficient at stimulating SF-1-dependent transcription. These observations suggest that oxysterols are key regulators of gene expression by binding to at least two members of the nuclear receptor family. The halfmaximal activity $\left(\mathrm{EC}_{50}\right)$ of 25-hydroxycholesterol stimulation of SF-1-dependent transcription is $5 \mu \mathrm{M}$, and therefore within the postulated physiological concentrations for oxysterols. Lala and colleagues (8) postulate that oxysterols generated through $\mathrm{P} 450 \mathrm{c} 27$ or as side products of other enzymatic activities could increase steroidogenesis by SF-1 activation. It is tempting at that point to speculate on a new signalling pathway controlling adrenal and gonadal activity!

\section{References}

1 Lala DS, Rice DA \& Parker KL. Steroidogenic factor 1, a key regulator of steroidogenic enzyme expression, is the mouse homolog of Fushi Tarazu-factor I. Molecular Endocrinology 1992 6 1249-1258. 
2 Morohashi K, Honda S, Inomata Y, Handa H \& Omura T. A common trans-acting factor, Ad4-binding protein, to the promoters of steroidogenic P-450s. Journal of Biological Chemistry $199226717913-17919$.

3 Cammas F, Pullinger G, Barker S \& Clarck A. The mouse adrenocorticotropin receptor gene: cloning and characterization of its promoter and evidence for a role for the orphan nuclear receptor steroidogenic factor 1. Molecular Endocrinology 199711 867-876.

4 Ingraham H, Lala D, Ikeda Y, Luo X, Shen W-H, Nachtigal NW, Abbud R, Nilsom JH \& Parker KL. The nuclear receptor steroidogenic factor 1 acts at multiple levels of the reproductive axis. Genes and Development 19948 2302-2312.

5 Luo X, Ikeda Y \& Parker KL. A cell-specific nuclear receptor is essential for adrenal and gonadal development and sexual differentiation. Cell 199477 481-490.
6 Shen W, Moore C, Ikeda Y, Parker K \& Ingraham H. Nuclear receptor steroidogenic factor 1 regulates Müllerian inhibiting substance gene expression: a link to the sex determination cascade. Cell 199477 651-661.

7 Parker K \& Schirmmer B. Steroidogenic factor 1: a key determinant of endocrine development and function. Endocrine Reviews 199718 361-377.

8 Lala DS, Syka P, Lazarchik S, Mangelsdorf D, Parker K \& Heyman R. Activation of the nuclear orphan receptor steroidogenic factor 1 by oxysterols. Proceedings of the National Academy of Sciences of the USA 199794 4895-4900.

9 Janowski B, Willy P, Devi T, Falck J \& Mangelsdorf D. An oxysterol signalling pathway mediated by the nuclear receptor LXRa. Nature 1996383 728-731. 